\title{
HEAPS study design: Health Effects of Air Pollution in Antwerp Schools
}

\author{
Evi Dons ${ }^{1,2}$, Martine Van Poppel ${ }^{1}$, S. De Prins ${ }^{1}$, Luc Int Panis ${ }^{1,2}$, G. Koppen ${ }^{1}$ \\ ${ }^{1}$ VITO - Flemish Institute for Technological Research, 2400 Mol, Belgium \\ ${ }^{2}$ Hasselt University, 3590 Diepenbeek, Belgium
}

Keywords: Black Carbon, UFP, traffic, health effects.

Presenting author email: martine.vanpoppel@vito.be

The HEAPS study (Health Effects of Air Pollution in Antwerp Schools) was set up to evaluate the health impact of traffic related air pollution on school children. Two research goals were defined in this study: (i) To evaluate which health effects can be measured in children in relation to traffic-related pollutants in their everyday surroundings.

(ii) To evaluate which traffic-related parameters are best for estimating exposures which may cause possible health effects.

The study set-up comprises two main parts: air pollution exposure assessment and health monitoring (Figure 1). Subtasks involved fixed and mobile air quality measurements, land use regression modelling, health assessment through questionnaires and noninvasive biomarker measurements. In this poster, the study design of the HEAPS project is discussed, and data are represented to provide evidence that the study design was appropriate.

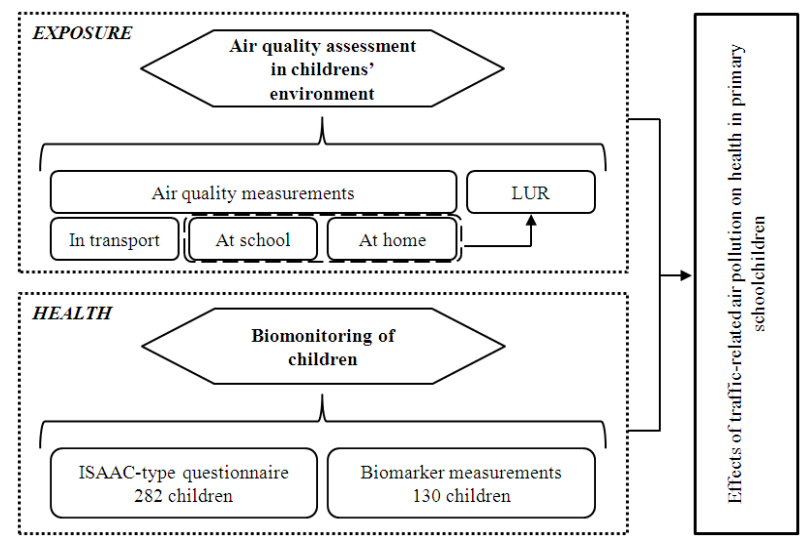

Figure 1. Study design of HEAPS

\section{Air pollution exposure assessment}

Air quality was monitored in two seasons, spring and autumn 2011, in 2 schools, at approximately 50 home locations and in transport (Table 1). The first school is located next to a quiet no-through road in the vicinity of a public park. The second school is situated next to a major road (>20,000veh/day). Air quality measurements were done in the playground at both schools, and at the street side for the school next to the busy road. Home measurements were done at selected home locations, at building facades or on street lighting at a height of $2-3 \mathrm{~m}$. Mobile measurements in two dominant transport modes per school were performed. Air pollution measurements were translated into exposure estimates in different time frames: a time frame to evaluate acute health effects, a time frame to assess subacute effects, and a time frame that considers long term health effects. UFP, PM, BC, NOx, and $\mathrm{O}_{3}$ concentrations measured at school the day before and on the day of medical examination were used for assessing acute health effects. To determine medium term exposure, personal exposure was estimated using modeled $\mathrm{BC}$ and $\mathrm{NO}_{2}$ concentrations from a land use regression (LUR) model. Time-activity patterns of children were taken into account by stratifying between exposure at home and at school. Transport from and to school is included as an additional micro-environment although these short trips were proven to be only of minor importance in aggregated exposure. Long term exposure was estimated at the home location using LUR models. Personal $\mathrm{NO}_{2}$ samplers were carried by the children during 1 week.

\begin{tabular}{lccc}
\hline \multicolumn{1}{c}{ Pollutant } & School & Home & Transport \\
\hline $\mathrm{PM}_{2.5}$ mass, filter (EC, OC) & $\mathrm{X}$ & & \\
$\mathrm{NO}_{2}$ & $\mathrm{X}$ & $\mathrm{X}$ & \\
Particle number & $\mathrm{X}$ & & $\mathrm{X}$ \\
Black carbon & $\mathrm{X}$ & $\mathrm{X}$ & $\mathrm{X}$ \\
PM factions, particle number & $\mathrm{X}$ & & \\
$\mathrm{NO}, \mathrm{NO}_{2}, \mathrm{NO}_{\mathrm{x}, \mathrm{O}_{3}}$ & $\mathrm{X}$ & & \\
\hline
\end{tabular}

Table 1. Overview of measured pollutants in different microenvironments.

\section{Health monitoring}

Health assessment consisted of a medical examination, non-invasive collection of biological matrices and oxidative stress, inflammation and cardiovascular biomarkers, and questionnaires. 130 children (aged 6-12) from 2 schools were selected for participation. Medical examinations were carried out in all 130 children in both spring and autumn of 2011, simultaneous with the air quality measurements. ISAACtype questionnaires were distributed amongst all children attending one of the schools.

Acknowledgement: the study was financed by the Flemish Environment Agency (VMM), the Flemish Administration of Environment, Nature and Energy (LNE), and the Flemish Agency for Care and Health (VZG). 